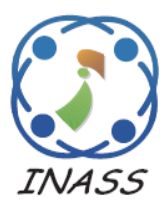

http://www.inass.org/

\title{
A Novel Denoising Algorithm Based on Superpixel Clustering and Dictionary Learning Approach
}

\author{
Jayapal Joseph ${ }^{1}$ \\ Ravi Subban ${ }^{2}$ \\ ${ }^{1}$ Bharathiar University, Coimbatore, India \\ ${ }^{I}$ Department of Computer Science and Engineering, Pondicherry University, Pondicherry, India \\ * Corresponding author’s Email: jayapal.bu@gmail.com
}

\begin{abstract}
Nowadays, the exploitation of images is inevitable in all the domains. However, the major issue being faced by the images is 'noise', which seriously affects the image quality. In order to address this issue, this article presents a novel denoising algorithm that is based on superpixel clustering and dictionary learning process. The superpixel clustering groups the similar pixels together to form a superpixel. As soon as the superpixels are computed, the sparse coefficients are computed by the steepest descent orthogonal matching pursuit algorithm. The process of dictionary updation is achieved by Discriminative K- Singular Value Decomposition (DK-SVD). The dictionary is trained by DK-SVD algorithm, as the discriminative capability of the algorithm is greater. The proposed approach shows promising results, which when compared to the existing techniques. Additionally, the time consumption of the proposed approach is very minimal than the comparative techniques.
\end{abstract}

Keywords: Image denoising, Superpixel clustering, Dictionary learning.

\section{Introduction}

Today's world flourishes with data and the images dominate all the other sorts of data. Images are intertwined with all the domains such as healthcare, remote sensing, robotics, pattern recognition and so on. Though the applicability of image processing is wider, it is susceptible to several issues such as poor image quality, noise, blurring effect, poor contrast and so on. Among all these issues, noise is the inevitable occurrence over images. Any image processing activity can yield better results, provided the images are noise-free. In order to minimize the effect of noise, a separate process namely 'noise removal' is required.

Image restoration is the process of recovering the original image from the damaged image $[1,2]$. The digital images are corrupted by means of several factors, which cannot be avoided. For instance, the digital images are corrupted during image acquisition, transmission or compression. On utilization of these corrupted images, the image processing activity cannot yield better results. Hence, the images are treated with the denoising process to eliminate the noise and thereby improving the image quality. However, the process of denoising is very challenging, as most of the denoising algorithms compromise with the original edges and textural patterns of an image. This results in the loss of information, which is unfavourable.

Hence, an effective denoising algorithm must preserve all the minute details of an image, such as edges and textural patterns. Inspite of the presence of numerous denoising algorithms in the literature, there is a constant demand for effective denoising algorithm. Taking this as a challenge, this article presents a denoising algorithm that relies on two important phases and they are superpixel clustering and sparse representation. The major purpose of superpixel clustering is to group relevant pixels together and these superpixels are again clustered. This step is followed by the construction of a dictionary and the sparse coefficients are upgraded iteratively. Finally, the image is reconstructed to form a noiseless image. The highlighting points of this article are summarized below. 
- The proposed approach produces better results, as the image is processed in both spatial and sparse based domain.

- The superpixel clustering algorithm clusters the relevant pixels by considering the image properties and the clustering operation does not stick on to a specific geometric structure.

- The DK-SVD algorithm, which is known for its representational ability is utilized for effective denoising.

- The incorporation of these two phases results in better noise removal.

- The exploitation of superpixel clustering and DK-SVD algorithm enhances the accuracy rate in detecting the sparse coefficients.

The remainder of this paper is organized as follows. Section 2 reviews the related literature with respect to image denoising process. The proposed work is elaborated in section 3 along with the general idea of the work. The performance of the proposed approach is evaluated in section 4. Finally, the concluding remarks of the proposed denoising algorithm are drawn in section 5 .

\section{Review of literature}

This section aims to review the related state-ofthe-art image denoising algorithms in the existing literature.

In [3], the images are denoised by applying curvelet transform, as curvelet transform can effectively represent the region and edge information. This work claims that the curvelet transform denoises the images effectively than wavelet transform, owing to its multiscale property. However, this work consumes more time. In [4], the images are denoised by a technique based on anisotropic diffusion model. The authors present a diffusion function which is based on Aubert model. The proposed technique proves better denoising results, while preserving the details of the image. However, the computational complexity of the work is more.

A hybrid algorithm to denoise CT images is presented in [5]. The proposed algorithm is the combination of Lucy-Richardson and non-local mean filter algorithms, which improves the image resolution and denoises the images. In [6], an image denoising algorithm for 3D sonar images is proposed. This algorithm is based on block matching technique and the denoising operation is done by ensemble average and transformation image processing. Both these works focus on a specific set of images and the applicability is restrained.
A denoising framework for images is presented in [7], which is based on light convolutional network. The light convolutional network is composed of three layers and they are high dimension projection, data completion and image reconstruction. This work proposes a weight loss function to enhance the learning speed of the algorithm. This work presents better denoising results with reasonable computational complexity. In [8], two different image restoration frameworks are proposed for noisy colour image and infrared image. This work proposes a scale map along with numerical solver functions to grasp minute details of an image. Additionally, multispectral shadow detection scheme is also utilized for improved performance. However, this approach consumes more time to achieve results.

In [9], a technique to denoise 1-D experimental signals by wavelet transform is proposed. The proposed technique allows the users to choose the degree of decomposition and the noise thresholds are computed without carrying out the process of noise estimation. The denoising method is applied over the approximation component and the noise thresholds can be adjusted. The proposed work is proven to be faster but the results are not convincing. A technique to remove noise from MR images is presented in [10]. This work is based on non-local means algorithm. This work utilizes wiener and median filters along with the non-local means filtering technique. However, the applicability of the work is limited.

A technique to eliminate non-white noise on hyperspectral images is presented in [11]. Initially, the hyperspectral images are treated with prewhitening procedure. This step is followed by the application of multidimensional wiener filtering for denoising the pre-whitened image. The signal is reconstructed by inverse pre-whitening procedure. This work focuses on a specific type of noise and involves computational overhead as well. In [12], a denoising algorithm is proposed for Optical Coherence Tomography (OCT) images, which incorporates both external and internal denoising procedures. Initially, the patches of the noisy image are compared with the patches of the image. All the noisy patches are denoised by the low rank technique and once the denoising process is over, the noiseless image is built by integrating all the noiseless patches.

A denoising approach for hyperspectral images is proposed in [13], which roots on Monte Carlo Sampling (MCS). This work considers both the spatial and spectral properties of images. Initially, a reference pixel is chosen in a random fashion and 
the similar pixels are chosen. The similar pixels are then manipulated with the posterior distribution and the noiseless pixel is fixed as the discrete conditional mean. This method preserves the image details and eliminates the noise effectively. Though the performance of the work is good, the computational and memory overhead of the work is high. In [14], a noise adjusted iterative low-rank matrix approximation is presented for denoising hyperspectral images. The clean signal and the noisy signal are segregated by means of an iterative regularization framework. An adaptive iterative factor selection technique is employed, as the noise level varies from band to band. This work consumes more time to arrive at the results.

The work proposed in [15] introduces a wiener filtering scheme that is approximated by graph fourier coefficients of the noisy image. This filter can either be applied to the entire image or to a specific patch of image. This work claims to show nominal Peak Signal to Noise Ratio (PSNR) value at the cost of memory overhead. In [16], a denoising method based on Morphological Component Analysis (MCA) is proposed. This method divides the image into two specific parts, which are texture and structure. The textural part employs All Phase Bio-orthogonal Transform (APBT) dictionary sparse representation is employed to denoise the textural part. The structural part of the image is denoised by means of block matching and 3D filtering technique. On clubbing these two parts, the final denoised image is obtained. This work produces better results but the time consumption of the work is more.

In [17], a denoising algorithm is presented which is based on non-local means algorithm. The nonlocal means algorithm is improvised by utilizing locality sensitivity hashing. This technique is proven to be faster and better performer in denoising. The denoising algorithm for mammogram images is presented in [18]. This algorithm employs gabor filter bank for removing noise from the mammogram images. A denoising method for furnace flame images is proposed in [19]. Mostly, the furnace flame images are affected by impulse and Gaussian noise. The proposed work deals with these noises by employing median filter and wavelet transform. Initially, adaptive median filter is applied to remove impulse noise, followed by which the image is decomposed by means of wavelet transform. Each band is processed with different filtering approach for effective denoising.

In [20], a technique for noise removal on multichannel images is presented. Initially, the spectral components are treated to extract linear features. This step is followed by the division of image into three components namely M-component and two gray scale images. The denoising procedure is applied over the gray scale images. This method preserves the image contrast however, this method is effective for hyperspectral images.

A denoising method to handle Gaussian, salt and pepper noise is presented in [21]. In this work, the images are divided into three parts namely texture, structure and edge by employing MCA algorithm. Median filter is utilized for noise removal in the texture part. This work shows better PSNR values. In [22], a denoising method that is based on adaptive dictionary being trained with noisy images is presented. The training process is carried out by $\mathrm{K}$ Singular Value Decomposition (K-SVD) algorithm along with steepest descent Orthogonal Matching Pursuit (OMP) algorithm.

In [23], a denoising technique is proposed for hyperspectral images, which is based on superpixel segmentation and low rank representation. Initially, the first principal component of hyspectral images is extracted by applying Principal Component Analysis (PCA). This is followed by the application of superpixel segmentation. This work claims that the mixed noises are successfully removed by this technique. In [24], a image denoising procedure is presented that is based on the superpixel segmentation. Initially, the images are segmented as superpixels by considering the geometrical structure. Similar patches of the superpixels are extracted, such that the images are denoised. This work claims that the computational complexity is reduced, however the denoising performance of the work is not upto the mark.

Though there are enormous denoising algorithms, most of the algorithms are image specific and involves computational complexity. Motivated by these existing works, this article intends to present a denoising algorithm that can preserve the image details, while proving better denoising results. The entire work is segregated into superpixel clustering and dictionary learning to achieve better performance. The following section elaborates the proposed denoising algorithm.

\section{Proposed denoising algorithm}

\subsection{General flow of the denoising algorithm}

The major goal of this article is to present a denoising algorithm that can effectively remove the noise being present in the image, while conserving the minute details of the image. The basic idea of the proposed denoising algorithm is to group related pixels by means of superpixel clustering algorithm. 


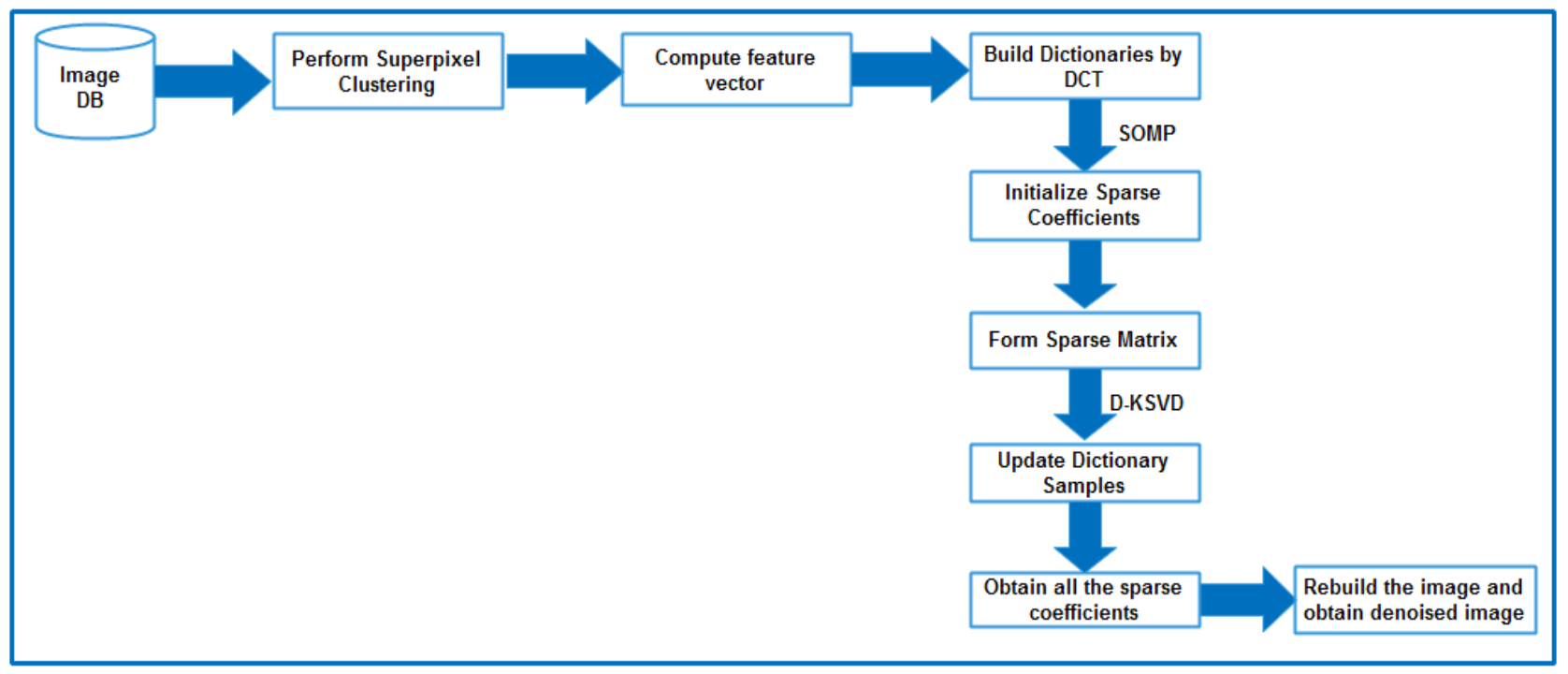

Figure.1 Overall flow of the proposed approach

This step is followed by segregating the image by steepest descent OMP algorithm, so as to obtain the sparse coefficients. The overall flow of the proposed approach is depicted in Fig. 1.

\subsection{Superpixel clustering algorithm}

The theme of superpixel clustering algorithm is to group similar pixels with each other. This pixel grouping is achieved by taking the characteristics of the pixels, such as texture, intensity, edges and so on. Hence, each superpixel consists of pixels, which are closely related to each other. Usual clustering algorithm follows some geometrical structure, which is not in the case of superpixel clustering algorithm. This means that the cluster operation can take any shape for grouping related pixels and the clustering algorithm is not restricted to any geometrical shapes.

Consider an image with $n o_{p}$ pixels and the number of superpixels is denoted as $n o_{s}$. The related pixels are grouped together and the resultant group is called as superpixel. The entire image is treated regionally, for which the size of the region has to be computed. The size of the region depends on the total number pixels and superpixels, which is represented as follows.

$$
R=\sqrt{n o_{p} / n o_{s}}
$$

The size of the region to select the centre pixel is computed by $2 R \times 2 R$. As soon as the size of the region is computed, the centre pixel is marked. This is followed by the random choice of pixel $p_{i}$ and is mapped with the nearest centroid of the cluster. The correlation between the pixels is measured by the following equation.

$$
\begin{aligned}
& \text { cor }=c s+\frac{\alpha}{R} \times d i s_{x y} \\
& c s=\sqrt{\left(c s_{a}-c s_{b}\right)^{2}} \\
& d i s_{x y}=\sqrt{\left(x_{a}-x_{b}\right)^{2}+\left(y_{a}-y_{b}\right)^{2}}
\end{aligned}
$$

In the above equations, $c s$ is the colour similarity and $\alpha$ is the variable that decides the conciseness of the superpixel. As the value of $\alpha$ is increased, the conciseness of the cluster is improved. This work fixes the value of $\alpha$ as 10 [25]. $R$ is the region, $c s_{a}, c s_{b}$ are the colour similarity of pixels $a$ and $b$. The $x$ and $y$ coordinates of pixel $a$ and $b$ are denoted by $x_{a}, x_{b}$ and $y_{a}, y_{b}$ respectively. Based on the pixel correlation, the similar pixels are grouped together, in order to form superpixels. This kind of superpixel clustering makes the denoising process easier, as the pixels within a superpixel are more related to each other.

As soon as the process of superpixel clustering is done, the feature vectors for each and every pixel within a superpixel are computed. The feature vector of a pixel comprises important features such as intensity and colour information and is represented as follows.

$$
f v=\left[c i, i n_{x}, i n_{y}, i n_{x x}, i n_{y y}\right]^{T}
$$

In the above equation, $c i$ is the colour information, $i n_{x}, i n_{y}, i n_{x x}, i n_{y y}$ are the first and second order derivatives of intensity in $x$ and $y$ axis. This step is followed by the computation of average feature vector of the superpixel and is denoted by the following equation. 


$$
f v_{\text {avg }}=\frac{1}{\mu} \sum_{i=1}^{\mu} f v_{i}
$$

In equation $6, \mu$ is the size of the superpixel and $f v_{i}$ is the feature vector of the pixel, which is a part of the superpixel. On successful completion of this process, the dictionary learning process is commenced and is presented below.

\subsection{Dictionary learning}

In this phase, the dictionary is constructed by means of Discrete Cosine Transform (DCT) redundant dictionary and the redundancy degree is set as 4 . The system is trained with the superpixels that are observed to be noisy by means of DK-SVD algorithm. At the end of this training process, the dictionary must be able to determine the quality of the superpixel. The sparse coefficients are computed by steepest descent orthogonal matching pursuit algorithm and the dictionary is updated by DK-SVD algorithm. The combination of DK-SVD and steepest descent orthogonal matching pursuit algorithm results in better denoising effect. The sparse representation of the superpixel is attained by the following equation.

$$
\min _{\delta}|| s p-D i c \delta \|_{2}^{2}+|| \delta||_{0}
$$

where $s p$ is the superpixel, $\delta_{s p}$ is the sparse coefficient of $s p, D i c_{s p}$ is the dictionary of $s p$ and $D i c_{s p} \in D I C$ and the index set $(Q)$ is set to null initially. The index set is udgraded in the next step by performing union operation. The upgradation of index set is represented as follows.

$$
q=\underset{q}{\operatorname{argmin}}\left\{\begin{array}{l}
\min \\
\delta_{s p}
\end{array}|| s p-D_{Q \cup(q)} \delta \|_{2}^{2}\right\}
$$

The index set $\mathrm{Q}$ is upgraded followed by the upgradation of the sparse coefficients of matrix $\delta_{s p}$. Subsequently, the sparse coefficients of $\delta_{s p}$ matrix are revised. This process is carried out for the pixels.

\subsection{Dictionary updation}

DK-SVD is the algorithm that modifies the dictionary by means of repetitive learning process and the dictionary is trained. The employed DKSVD algorithm takes the multiple features of the noisy image into account for dictionary construction. This improves the performance of the proposed work with effective denoising. The reason for the inclusion of DK-SVD algorithm in the place of KSVD algorithm is its discrimination ability of noisy pixels. Additionally, the DK-SVD algorithm is originally meant for noiseless image, however the proposed work modifies the algorithm such that it works for noisy images too. The process of dictionary updation is given below.

$$
\begin{gathered}
\left\{\delta_{a b}, D i c, S P\right\}=\underset{\operatorname{Dic}, \delta_{a b}, s p}{\operatorname{argmin}}\left(\gamma\left\|S P_{i 1}-S P_{i 2}\right\|_{2}^{2}+\right. \\
\left.\sum_{a b} \rho_{a b}\left\|\delta_{a b}\right\|_{0}+\sum_{a b} \mid\left\|\oplus \delta_{a b}-S P_{i j} S P\right\|_{2}^{2}\right)
\end{gathered}
$$

In the above equation, $\delta_{a b}$ is the sparsity coefficient, Dic is the dictionary and $S P$ is the superpixel. Here, $\gamma$ is the lagrange multiplier and the first part of the equation measures the distance between the two superpixels, where $S P_{i 1}$ is the noise estimated superpixel and $S P_{i 1}$ is the denoised superpixel. The second part of the equation denotes the constraints of sparsity. The final part of the equation deals with the representation of the image rebuilding step. $\oplus \delta_{a b}$ is the estimated image after reconstruction. The complete algorithm of the proposed approach is presented below.

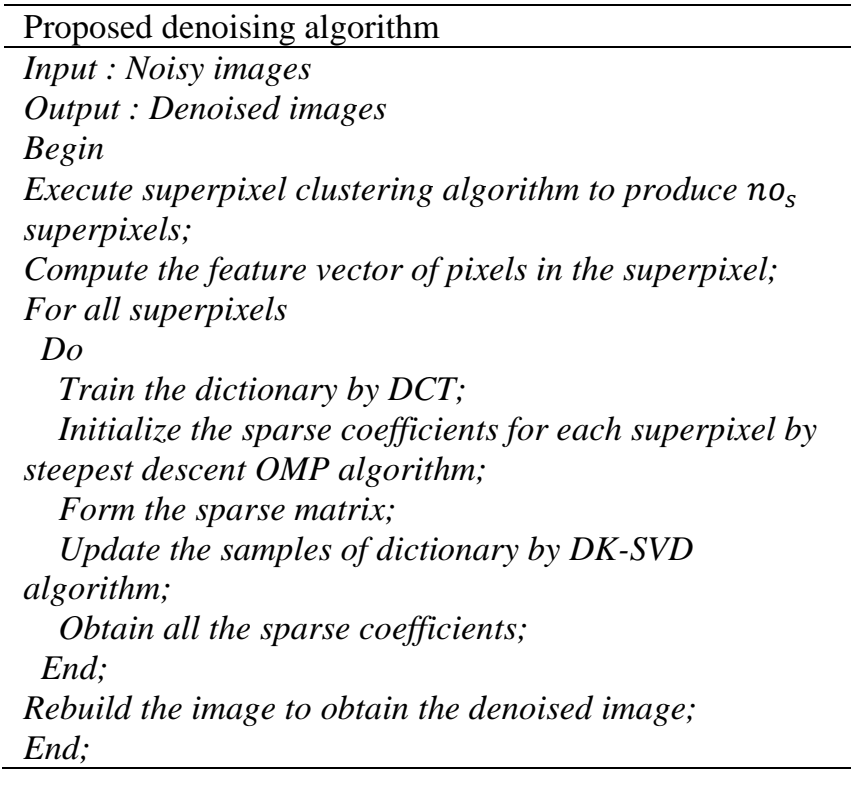

The entire process of dictionary updation is summarized as follows. Initially, the dictionary is initialized and the superpixels are passed into the system. For each and every superpixel, the steepest descent orthogonal matching pursuit algorithm is utilized to manipulate the representative vector. The sparse matrix is formed and the samples of the dictionary are updated as follows. The sparse coefficients whose value is not zero is picked out by

$$
n s_{s p}=\left\{[a, b] \delta_{a b}(s p) \neq 0\right\}
$$


For each sample and the coefficients, the representative fault is then computed by

$$
\begin{gathered}
\left(\operatorname{dic}_{i}, \delta_{i}\right)=\underset{\operatorname{dic}_{i}, \delta_{i}}{\operatorname{argmin}} \operatorname{dic}_{i}|| r f_{i}-\operatorname{dic}_{i} \times\left.\delta_{i}\right|_{F} \\
r f_{i}=T S-\sum_{p \neq i} \operatorname{dic}_{p} \times \delta_{p}
\end{gathered}
$$

where $d i c_{i}$ is the $i^{\text {th }}$ sample of the dictionary and its corresponding sparse coefficient is represented by $\delta_{i}$. $r f_{i}$ is the representative fault of the $i^{\text {th }}$ sample and $F$ stands for Frobenius transformation. In equation $12, T S$ is the training sample. Finally, the denoised image is obtained by

$$
\begin{array}{rr}
D I=\left(\gamma+\sum_{a b} s p_{a b}^{d e g}+s p_{a b}\right)^{-1} & \left(\gamma S P_{i 2}+\right. \\
\left.\sum_{a b} s p_{a b}^{d e g} D i c \delta_{a b}\right) & \text { (13) }
\end{array}
$$

deg indicates the repeated presence of the sample, that is when the degree is minimal then the occurrence of that particular sample is minimal. Thus, the final noise-free image is constructed by summing up all the superpixels.

\section{Results and discussion}

The performance of the proposed algorithm is evaluated in terms of Peak Signal to Noise Ratio (PSNR), Figure of Merit (FoM) and Feature Similarity Index [26]. PSNR is the widely employed qualitative measure for assessing the quality of an image. The FSIM is the recently proposed qualitative measure that computes the resemblance between two images. The resemblance between the images is computed by clubbing the phase congruency and gradient magnitude features. As far as the quality measures PSNR and FSIM are considered, FSIM is easily perceived by human eyes and the PSNR is hard to observe. The FoM compares the edge information between the original and noisy images. The white Gaussian noise is added to the images in different degrees such as 30 , 50 and 70. This work is simulated on Matlab environment in a standalone system with $4 \mathrm{~GB}$ RAM. The performance of the proposed approach is compared with NLM filter [27], K-SVD [28] and SAIST [29]. The experimental results of the proposed approach and all the analogous techniques for varying noise level are tabulated in Table 1.

From the above table, it can be noticed that the proposed approach works well with respect to PSNR, FSIM and FoM, when compared to the comparative techniques. The PSNR value of the proposed approach is greater than all the comparative techniques in all the three cases. The average FSIM value of SAIST is greater than the proposed approach, when the noise variance is 70 . However, the difference between SAIST and the proposed approach is very minimal. When the noise variance is set as 50, the SAIST shows better FoM value and the value difference between the SAIST and the proposed approach is just 0.014 .

It can be observed that the greater noise variance shows direct impact over the PSNR value. When the noise variance is set as 30, the PSNR value is 30.529 . As the noise variance is increased to 50 and 70 , the PSNR value diminishes to 25.5 and 24.55 respectively. Similarly, the FSIM and FoM values are also found to be decreasing, as the noise variance is increased. The following figure depicts the sample visual results of the proposed approach.

From the experimental results, it is evident that the proposed approach can deal with the noise effectively. The better denoising results are achieved for the sample images, as the proposed work involves superpixel clustering followed by the dictionary learning process. The results show that the proposed approach denoises the images effectively, while conserving the details of the images. This statement is justified by incorporating the FoM metric, which is meant for checking the consistency of the edge information. Additionally, the time complexity of the proposed approach is compared with the analogous techniques and is depicted in Fig. 4.

The time consumption analysis is carried out by selecting ten benchmark images. The average time consumption for all the ten images is computed and the graph is plotted. On average, NLM consumes more time than the other techniques and it consumes 987 seconds. K-SVD follows NLM with 406 seconds for the same set of images. The SAIST technique requires 206 seconds to denoise the ten images. On comparison, the proposed approach consumes the least time to carry out the denoising process and it consumes 184 seconds. The reason for the minimal time consumption is the inclusion of superpixel clustering and the dictionary learning procedure.

Additionally, the performance of the proposed approach is compared with the existing techniques such as SS-LRR [23] and SS-NLM [24] in terms of Peak-Signal-to-Noise Ratio (PSNR). The below presented figure depicts the average PSNR value of the proposed and the existing techniques.

The PSNR value determines the quality of an image. The greater the PSNR value, the better is the quality of an image. Fig. 5 presents the average PSNR value of different images by varying the 
Table 1. Comparative analysis on PSNR (dB), FSIM and FoM values

\begin{tabular}{|c|c|c|c|c|}
\hline \multirow{2}{*}{ Images } & \multicolumn{4}{|c|}{$\sigma=30$} \\
\hline & NLM & K-SVD & SAIST & Proposed approach \\
\hline \multirow{3}{*}{ Baboon } & 23.08 & 25.96 & 27.32 & 27.86 \\
\hline & 0.926 & 0.919 & 0.923 & 0.953 \\
\hline & 0.486 & 0.693 & 0.702 & 0.748 \\
\hline \multirow{3}{*}{ Barbara } & 25.6 & 27.3 & 27.7 & 28.3 \\
\hline & 0.932 & 0.942 & 0.950 & 0.958 \\
\hline & 0.581 & 0.623 & 0.682 & 0.734 \\
\hline \multirow{3}{*}{ Scenary } & 28.3 & 30.86 & 31.43 & 31.62 \\
\hline & 0.921 & 0.905 & 0.950 & 0.952 \\
\hline & 0.607 & 0.721 & 0.743 & 0.768 \\
\hline \multirow{3}{*}{ Satellite image } & 26.48 & 28.8 & 29.32 & 29.45 \\
\hline & 0.927 & 0.907 & 0.916 & 0.922 \\
\hline & 0.812 & 0.832 & 0.887 & 0.914 \\
\hline \multirow{3}{*}{ Boat } & 27.43 & 31.36 & 31.59 & 32.36 \\
\hline & 0.923 & 0.912 & 0.931 & 0.924 \\
\hline & 0.426 & 0.642 & 0.725 & 0.717 \\
\hline \multirow{3}{*}{ Waterfall } & 28.32 & 32.43 & 32.93 & 32.96 \\
\hline & 0.931 & 0.911 & 0.936 & 0.934 \\
\hline & 0.584 & 0.732 & 0.802 & 0.824 \\
\hline \multirow{3}{*}{ MRI } & 26.32 & 29.32 & 30.21 & 30.18 \\
\hline & 0.962 & 0.964 & 0.970 & 0.976 \\
\hline & 0.623 & 0.734 & 0.802 & 0.823 \\
\hline \multirow{3}{*}{ Building } & 29.05 & 32.36 & 32.98 & 33.05 \\
\hline & 0.965 & 0.964 & 0.969 & 0.971 \\
\hline & 0.613 & 0.714 & 0.702 & 0.713 \\
\hline \multirow{3}{*}{ Tree } & 26.43 & 29.31 & 29.81 & 29.83 \\
\hline & 0.932 & 0.923 & 0.937 & 0.929 \\
\hline & 0.328 & 0.643 & 0.694 & 0.673 \\
\hline \multirow{3}{*}{ Lake } & 26.14 & 29.05 & 29.32 & 29.68 \\
\hline & 0.943 & 0.936 & 0.943 & 0.947 \\
\hline & 0.459 & 0.643 & 0.754 & 0.782 \\
\hline \multirow{3}{*}{ Average } & 26.715 & 29.675 & 30.261 & 30.529 \\
\hline & 0.936 & 0.928 & 0.942 & 0.946 \\
\hline & 0.551 & 0.697 & 0.749 & 0.769 \\
\hline \multirow{2}{*}{ Images } & \multicolumn{4}{|c|}{$\sigma=50$} \\
\hline & NLM & K-SVD & SAIST & Proposed approach \\
\hline \multirow{3}{*}{ Baboon } & 20.96 & 22.03 & 22.43 & 22.73 \\
\hline & 0.932 & 0.926 & 0.947 & 0.942 \\
\hline & 0.283 & 0.548 & 0.586 & 0.579 \\
\hline \multirow{3}{*}{ Barbara } & 17.93 & 21.8 & 22.65 & 23.86 \\
\hline & 0.934 & 0.923 & 0.938 & 0.940 \\
\hline & 0.536 & 0.632 & 0.581 & 0.656 \\
\hline \multirow{3}{*}{ Scenary } & 23.3 & 26.04 & 26.34 & 27.43 \\
\hline & 0.904 & 0.883 & 0.909 & 0.902 \\
\hline & 0.473 & 0.618 & 0.714 & 0.698 \\
\hline & 20.8 & 24.63 & 24.72 & 24.78 \\
\hline Satellite image & 0.908 & 0.889 & 0.896 & 0.903 \\
\hline & 0.689 & 0.814 & 0.856 & 0.812 \\
\hline & 24.2 & 26.84 & 27.43 & 28.03 \\
\hline Boat & 0.913 & 0.884 & 0.903 & 0.908 \\
\hline & 0.329 & 0.483 & 0.612 & 0.578 \\
\hline & 23.61 & 26.73 & 27.84 & 28.58 \\
\hline Waterfall & 0.952 & 0.948 & 0.959 & 0.957 \\
\hline & 0.484 & 0.672 & 0.703 & 0.723 \\
\hline & 21.52 & 25.08 & 25.83 & 25.38 \\
\hline MRI & 0.918 & 0.893 & 0.897 & 0.905 \\
\hline & 0.462 & 0.658 & 0.744 & 0.692 \\
\hline
\end{tabular}




\begin{tabular}{|c|c|c|c|c|}
\hline Building & $\begin{array}{c}18.3 \\
0.927 \\
0.529\end{array}$ & $\begin{array}{l}20.43 \\
0.925 \\
0.556\end{array}$ & $\begin{array}{l}20.94 \\
0.921 \\
0.554\end{array}$ & $\begin{array}{l}21.43 \\
0.935 \\
0.593\end{array}$ \\
\hline Tree & $\begin{array}{c}23.7 \\
0.834 \\
0.283\end{array}$ & $\begin{array}{l}25.62 \\
0.873 \\
0.449\end{array}$ & $\begin{array}{l}26.23 \\
0.867 \\
\mathbf{0 . 5 6 3}\end{array}$ & $\begin{array}{l}\mathbf{2 6 . 3 2} \\
\mathbf{0 . 8 9 8} \\
0.534\end{array}$ \\
\hline Lake & $\begin{array}{c}23.5 \\
0.934 \\
0.355\end{array}$ & $\begin{array}{l}25.38 \\
0.935 \\
0.504\end{array}$ & $\begin{array}{l}26.42 \\
0.928 \\
\mathbf{0 . 6 8 2}\end{array}$ & $\begin{array}{l}\mathbf{2 6 . 5 1} \\
\mathbf{0 . 9 4 6} \\
0.594\end{array}$ \\
\hline Average & $\begin{array}{l}21.78 \\
0.915 \\
0.442\end{array}$ & $\begin{array}{l}24.45 \\
0.907 \\
0.593\end{array}$ & $\begin{array}{l}25.08 \\
0.914 \\
\mathbf{0 . 6 5 9}\end{array}$ & $\begin{array}{c}\mathbf{2 5 . 5} \\
\mathbf{0 . 9 2 3} \\
0.645\end{array}$ \\
\hline Images & \multicolumn{4}{|c|}{$\sigma=70$} \\
\hline Images & NLM & K-SVD & SAIST & Proposed approach \\
\hline Baboon & $\begin{array}{l}20.63 \\
0.918 \\
0.234\end{array}$ & $\begin{array}{l}21.32 \\
0.914 \\
0.226\end{array}$ & $\begin{array}{l}21.72 \\
0.924 \\
0.283 \\
\end{array}$ & $\begin{array}{l}22.34 \\
0.928 \\
0.292\end{array}$ \\
\hline Barbara & $\begin{array}{l}17.82 \\
0.914 \\
0.431\end{array}$ & $\begin{array}{l}19.58 \\
0.904 \\
0.437\end{array}$ & $\begin{array}{l}21.12 \\
0.921 \\
0.386\end{array}$ & $\begin{array}{l}22.48 \\
0.929 \\
0.497\end{array}$ \\
\hline Scenary & $\begin{array}{l}22.03 \\
0.886 \\
0.324\end{array}$ & $\begin{array}{l}24.23 \\
0.862 \\
0.356\end{array}$ & $\begin{array}{l}25.64 \\
\mathbf{0 . 8 9 4} \\
\mathbf{0 . 5 5 2}\end{array}$ & $\begin{array}{l}\mathbf{2 5 . 6 7} \\
0.882 \\
0.549\end{array}$ \\
\hline Satellite image & $\begin{array}{l}22.56 \\
0.889 \\
0.494\end{array}$ & $\begin{array}{l}22.53 \\
0.867 \\
0.623\end{array}$ & $\begin{array}{l}23.45 \\
0.894 \\
0.726\end{array}$ & $\begin{array}{l}23.43 \\
0.889 \\
0.693\end{array}$ \\
\hline Boat & $\begin{array}{l}23.47 \\
\mathbf{0 . 8 9 3} \\
0.283\end{array}$ & $\begin{array}{l}25.42 \\
0.854 \\
0.294\end{array}$ & $\begin{array}{l}26.19 \\
0.892 \\
\mathbf{0 . 4 1 6}\end{array}$ & $\begin{array}{l}\mathbf{2 6 . 7 4} \\
0.889 \\
0.398\end{array}$ \\
\hline Waterfall & $\begin{array}{l}22.32 \\
\mathbf{0 . 9 4 7} \\
0.338\end{array}$ & $\begin{array}{l}24.53 \\
0.924 \\
0.356\end{array}$ & $\begin{array}{l}26.43 \\
0.946 \\
\mathbf{0 . 5 5 9}\end{array}$ & $\begin{array}{l}\mathbf{2 6 . 8 9} \\
0.942 \\
0.542\end{array}$ \\
\hline MRI & $\begin{array}{l}19.82 \\
\mathbf{0 . 8 9 6} \\
0.372\end{array}$ & $\begin{array}{l}22.86 \\
0.871 \\
0.412\end{array}$ & $\begin{array}{l}26.42 \\
0.892 \\
\mathbf{0 . 5 8 3}\end{array}$ & $\begin{array}{l}\mathbf{2 6 . 9 8} \\
0.889 \\
0.576\end{array}$ \\
\hline Building & $\begin{array}{l}18.31 \\
0.919 \\
0.361 \\
\end{array}$ & $\begin{array}{l}19.36 \\
0.917 \\
0.379\end{array}$ & $\begin{array}{l}19.49 \\
0.918 \\
0.334\end{array}$ & $\begin{array}{l}20.19 \\
0.926 \\
0.442\end{array}$ \\
\hline Tree & $\begin{array}{l}22.68 \\
\mathbf{0 . 8 8 2} \\
0.198 \\
\end{array}$ & $\begin{array}{l}24.52 \\
0.834 \\
0.215\end{array}$ & $\begin{array}{l}25.03 \\
0.876 \\
0.336\end{array}$ & $\begin{array}{l}\mathbf{2 5 . 2 9} \\
0.865 \\
\mathbf{0 . 3 3 9}\end{array}$ \\
\hline Lake & $\begin{array}{l}22.53 \\
0.913 \\
0.293\end{array}$ & $\begin{array}{l}24.48 \\
0.912 \\
0.268\end{array}$ & $\begin{array}{l}25.38 \\
0.926 \\
\mathbf{0 . 4 1 9}\end{array}$ & $\begin{array}{l}\mathbf{2 5 . 5 2} \\
\mathbf{0 . 9 3 5} \\
0.399\end{array}$ \\
\hline Average & $\begin{array}{l}21.21 \\
0.905 \\
0.332\end{array}$ & $\begin{array}{l}22.88 \\
0.885 \\
0.356\end{array}$ & $\begin{array}{l}24.08 \\
\mathbf{0 . 9 0 8} \\
0.459\end{array}$ & $\begin{array}{l}\mathbf{2 4 . 5 5} \\
0.907 \\
\mathbf{0 . 4 7 2}\end{array}$ \\
\hline
\end{tabular}

value of $\sigma$. As the value of $\sigma$ increases, the PSNR value decreases. However, the denoising techniques should prove convincing quality. The PSNR value of SS-NLM is observed to be lower when compared to SS-LRR and the proposed approach. The reason is that this technique focusses on reducing the computational complexity and not the image quality. SS-LRR works on par with the proposed approach. However, the proposed approach proves better PSNR than SS-LRR. The reason is the construction of dictionary with updation and the employment of superpixel clustering. The maximum PSNR value attained by SS-NLM is 27.86. The PSNR value shown by SS-LRR and the proposed approach are 28.38 and 32.27 respectively. The proposed approach shows considerable difference in PSNR value against SS-NLM and SS-LRR. Thus, the proposed approach consumes minimal eriod of time to generate better denoised images. 


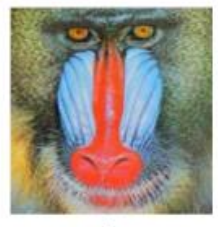

a

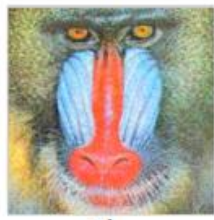

a1

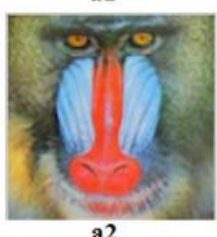

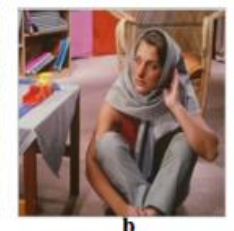

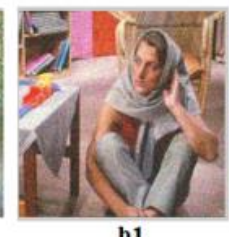

b1

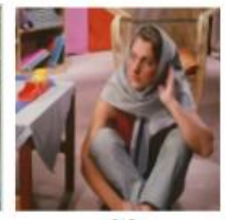

b2
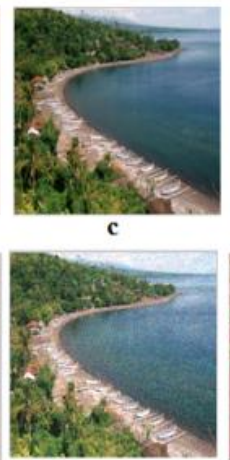

c1

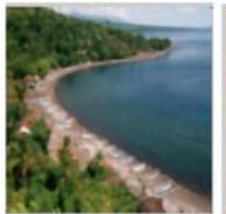

c2
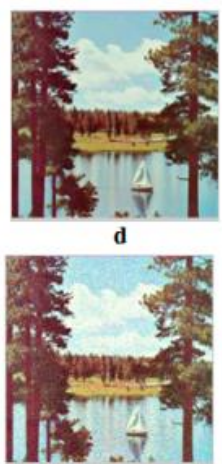

d1

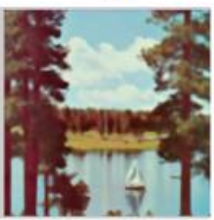

d2

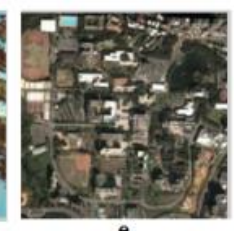

e

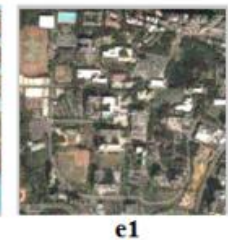

e1

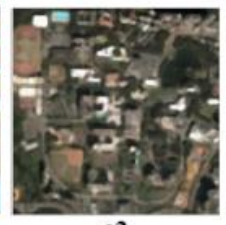

e2
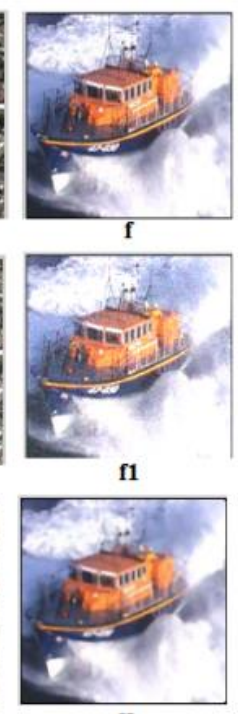

Figure.1 a-f are the original images, a1-f1 are the noisy images, a2-f2 are the denoised images $(\sigma=30)$
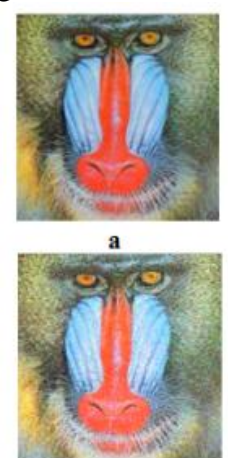

a1

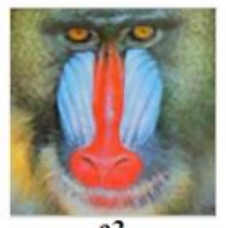

a2
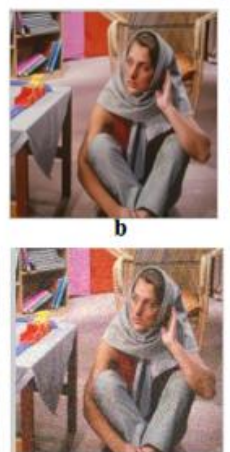

b1

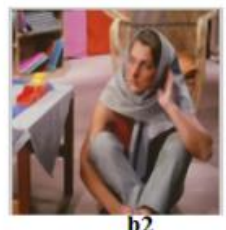

(2)
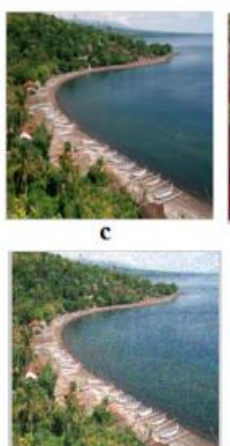

c1

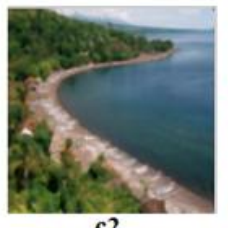

c2
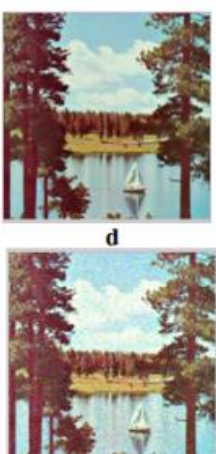

d1

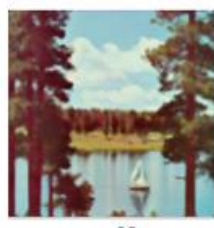

d2
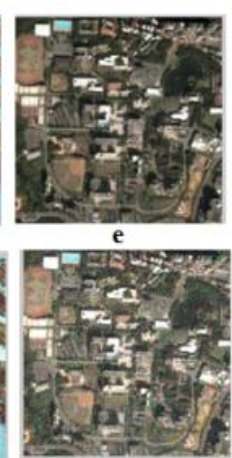

e1

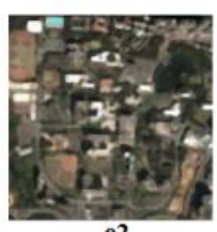

e2
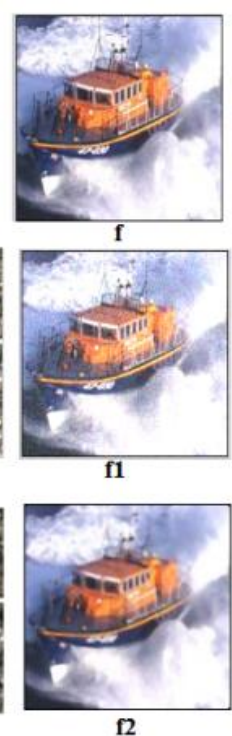

Figure. 2 a-f are the original images, a1-f1 are the noisy images, a2-f2 are the denoised images $(\sigma=50)$

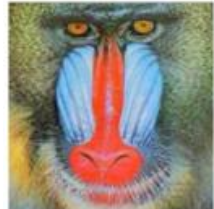

a

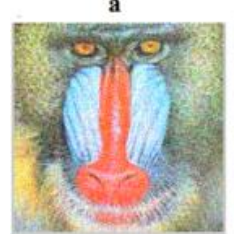

a1
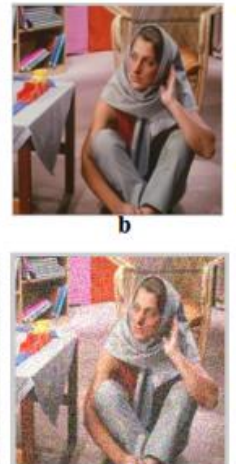

b1

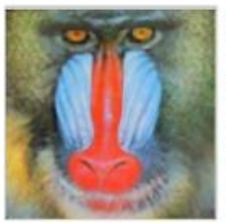

2

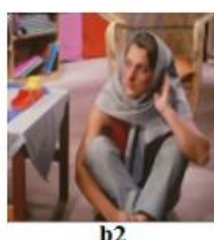

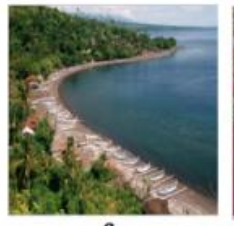

c

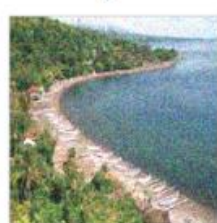

c1
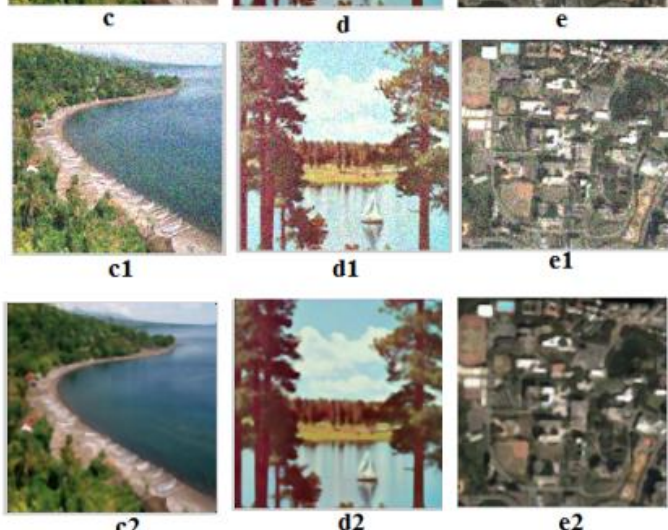

e

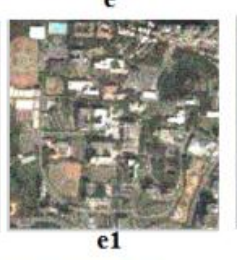

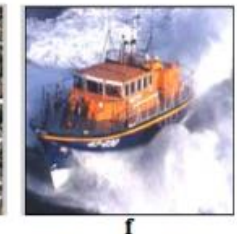
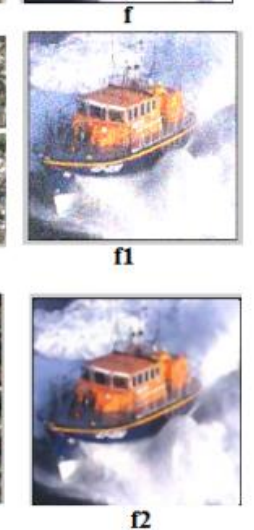

Figure. 3 a-f are the original images, a1-f1 are the noisy images, a2-f2 are the denoised images $(\sigma=70)$ 


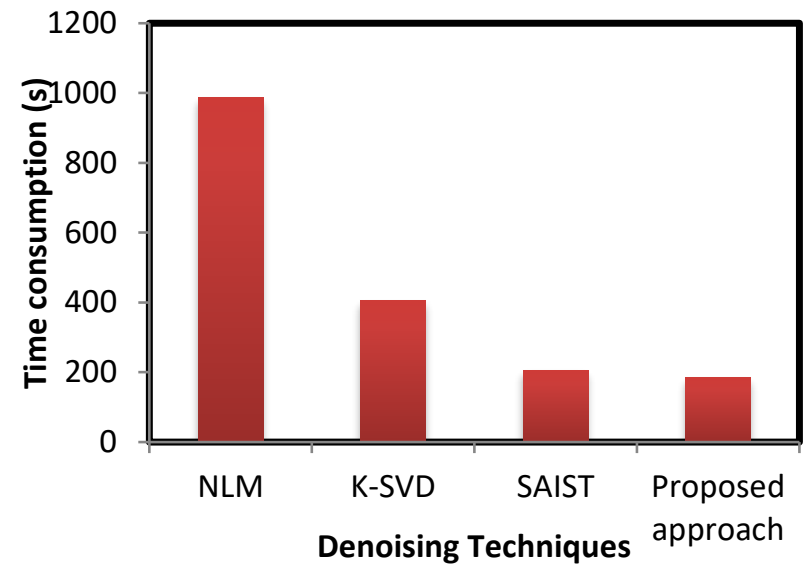

Figure.4 Comparative analysis on time consumption

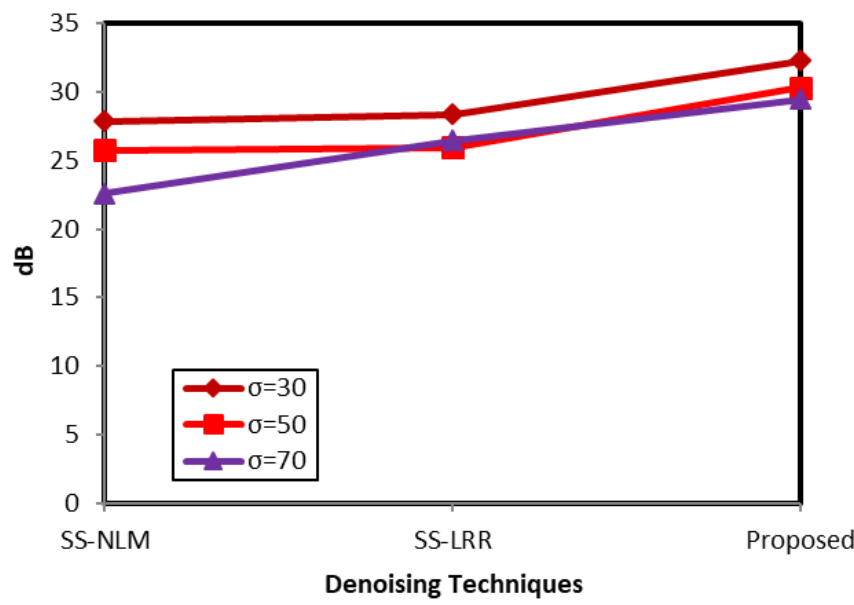

Figure.5 PSNR analysis

\section{Conclusion}

This article presents a simple yet an efficient algorithm to denoise the images. The proposed approach relies on two functional blocks which are superpixel clustering and dictionary learning. The superpixel clustering process clusters the similar pixels together to construct a superpixel. The feature vectors are computed for each and every pixel in the superpixel and the correlation between the superpixels are found out. The sparse coefficients are then computed by means of steepest descent OMP algorithm. The dictionary is updated by means of DK-SVD algorithm, owing to its discriminating ability. Finally, the denoised image is obtained and the quality of the denoised images is measured by standard performance metrics such as PSNR, FSIM and FoM. The results attained by the proposed approach are compared with the existing denoising techniques and the proposed work outperforms the analogous techniques. Additionally, the time consumption of the proposed approach is measured, which is minimal than the existing techniques. In future, this work plans to address the issue of video denoising.

\section{References}

[1] A. Rosenfeld and A. C. Kak, "Digital Picture Processing." Second Edition, Academic Press, Orlando, USA, 1982.

[2] C. Carl, "Regularization in image restoration and reconstruction," Handbook of Image and Video Processing, Academic Press, San Diego, pp. 141-160, 2005.

[3] Q. Yi, Y. Weng, and J. He, "Image denoise based on Curvelet transform", In: Proc. of IEEE Workshop on Electronics, Computer and Applications, Ottawa, Canada, 8-9 May 2014.

[4] P. Yang and Z. Chang, "An improved image anisotropic diffusion denoise model", In: Proc of IET International Conference on Information Science and Control Engineering, Shenzhen, China, 7-9 Dec, 2012.

[5] W. Shao, J. Ni, and C. Zhu, "A Hybrid Method of Image Restoration and Denoise of CT Images", In: Proc of International Conference on Internet Computing for Science and Engineering, Henan, China, 21-23 April, 2012.

[6] W. Cong and J. Lu, "An improved threedimensional block matching image de-noise algorithm and its application in a $3 \mathrm{D}$ imaging sonar", In: Proc of IEEE Ocean Acoustics, Harbin, China, 9-11 Jan, 2016.

[7] X. Zhang and R. Wu, "Fast depth image denoising and enhancement using a deep convolutional network", In: Proc. of IEEE Conference on Acoustics, Speech and Signal Processing, Shanghai, China, 20-25 March, 2016.

[8] X. Shen, Q. Yan, L. Xu, L. Ma, and J. Jia, "Multispectral Joint Image Restoration via Optimizing a Scale Map", IEEE Transactions on Pattern Analysis and Machine Intelligence, Vol.37, No.12, pp.2518-2530, 2015.

[9] M. Srivastava, C. L. Anderson, and J. H. Freed, "A New Wavelet Denoising Method for Selecting Decomposition Levels and Noise Thresholds", IEEE Access, Vol.4, pp. 38623877, 2016.

[10] N. Joshi, S. Jain, and A. Agarwal, "An improved approach for denoising MRI using non local means filter", In: Proc of International Conference on Next Generation Computing Technologies, Dehradun, India, 1416 Oct, 2016.

[11] X. Liu, Y. Feng, Y. Li, X. Liu, W. Zhao, and M. $\mathrm{Fu}$, "Denoising hyperspectral images with non- 
white noise based on tensor decomposition", In: Proc of IEEE International Conference on Digital Signal Processing, Beijing, China, 1618 Oct, 2016.

[12] X. Liu, Z. Yang, and J. Wang, "A novel noise reduction method for optical coherence tomography images", In: Proc. of International Congress on Image and Signal Processing, BioMedical Engineering and Informatics, Datong, China, 15-17 Oct. 2016.

[13] L. Xu, F. Li, A. Wong, and D. A. Clausi, "Hyperspectral Image Denoising Using a Spatial-Spectral Monte Carlo Sampling Approach", IEEE Journal of Selected Topics in Applied Earth Observations and Remote Sensing, Vol.8, No.6, pp. 3025-3038, 2015.

[14] W. He, H. Zhang, L. Zhang, and H. Shen, "Hyperspectral Image Denoising via NoiseAdjusted Iterative Low-Rank Matrix Approximation", IEEE Journal of Selected Topics in Applied Earth Observations and Remote Sensing, Vol.8, No.6, pp. 3050-3061, 2015.

[15] A. C. Yagan, M. T. Özgen, "A spectral graph wiener filter in graph fourier domain for improved image denoising", In: Proc. of IEEE Global Conference on Signal and Information Processing, Washington DC, USA, 7-9 Dec, 2016.

[16] Y. Cheng and Z. Liu, "Image Denoising Algorithm Based on Structure and Texture Part", In: Proc. of International Conference on Computational Intelligence and Security, Wuxi, China, 16-19 Dec 2016.

[17] N. Divakar and R. V. Babu, "Denoising in a Jiffy: A fast and GPU friendly algorithm for image denoising", In: Proc. of International Conference on Signal Processing and Communications, Bangalore, India, 12-15 Jun 2016.

[18] V. Vedanarayanan and N. M. Nandhitha, "Suitability of Gabor filter for denoising and contrast enhancement of digital mammograms", In: Proc. of International Conference on Science Technology Engineering and Management, Chennai, India, 30-31 Mar, 2016.

[19] A. JingYu and M. XianMin, "A New Method of Image Denoising for Furnace Flame", In: Proc. of International Symposium on Computer, Consumer and Control, Xi'an, China, 4-6 July, 2016.

[20] M. Rizkinia, T. Baba, K. Shirai, and M. Okuda, "Local Spectral Component Decomposition for Multi-Channel Image Denoising", IEEE
Transactions on Image Processing, Vol.25, No.7, pp. 3208-3218, 2016.

[21] X. Deng and Z. Liu, "An Improved Image Denoising Method Applied in Resisting Mixed Noise Based on MCA and Median Filter", In: Proc. of International Conference on Computational Intelligence and Security, Shenzhen, China, 19-20 Dec, 2015.

[22] X. Deng and Z. Liu, "Image denoising based on steepest descent OMP and K-SVD", In: Proc of IEEE International Conference on Signal Processing, Communications and Computing, Ningbo, China, 19-22 Sep, 2015.

[23] J. Ma, C. Li, Y. Ma, and Z. Wang, "Hyperspectral image denoising based on lowrank representation and superpixel segmentation", In: Proc. of IEEE International Conference on Image Processing, 25-28 Sept, Phoenix, USA, 2016.

[24] W. Liu and S. Wu, "Superpixels-based nonlocal means image denoising", In: Proc. of IEEE 11th Conference on Industrial Electronics and Applications, 5-7 June, Hefei, China, 2016.

[25] R. Achanta, A. Shaji, K. Smith, A. Lucchi, P. Fua, and S. Susstrunk, "SLIC Superpixels", EPFL Technical Report No. 149300; EPFL: Lausanne, Switzerland, 2010.

[26] L. Zhang, D. Zhang, X. Mou, and D. Zhang, "FSIM: A feature similarity index for image quality assessment," IEEE Trans. Image Process., Vol. 20, No. 8, pp. 2378-2386, Aug. 2011.

[27] A. Buades, B. Coll, and J.M. Morel, "A nonlocal algorithm for image denoising", In: Proc. of IEEE Computer Society Conference on Computer Vision and Pattern Recognition, San Diego, CA, USA, 20-25 Jun, 2005.

[28] M. Elad and M. Aharon, "Image denoising via sparse and redundant representations over learned dictionaries", IEEE Transactions on Image Processing, Vol. 15, No. 12, pp. 37363745, Dec. 2006.

[29] W. Dong, G. Shi, and X. Li, "Nonlocal image restoration with bilateral variance estimation: A low-rank approach", IEEE Transactions on Image Processing, Vol. 22, No. 2, pp. 700-711, Feb. 2013. 